\title{
Racial Differences in Prevalence and Treatment for Psoriatic Arthritis and Ankylosing Spondylitis by Insurance Coverage in the USA
}

\author{
Alexis Ogdie · Wesley Matthias • Richard J. Thielen • Daniel Chin • \\ Christopher D. Saffore
}

Received: July 21, 2021 / Accepted: September 1, 2021 / Published online: September 25, 2021

(C) The Author(s) 2021

\section{ABSTRACT}

Introduction: Patients with psoriatic arthritis (PsA) and ankylosing spondylitis (AS) may receive suboptimal care, and differences in care by race/ethnicity, sex, and insurance coverage are not well studied.

Methods: This was a descriptive, retrospective cross-sectional US claims database analysis utilizing the Medicaid multi-state segment of the IBM ${ }^{\circledR}$ MarketScan ${ }^{\circledR}$ Commercial Claims and Encounters Supplemental Database and Optum Insight Clinformatics ${ }^{\circledR}$ Data Mart database for 2019. Patients aged $\geq 18$ years with PsA or AS and continuous medical and pharmacy coverage were included. Outcomes evaluated were prevalence and percentage of patients receiving biologic disease-modifying antirheumatic drugs (bDMARDs)/targeted synthetic DMARDs

Supplementary Information The online version contains supplementary material available at https:// doi.org/10.1007/s40744-021-00370-4.

\footnotetext{
A. Ogdie

University of Pennsylvania, Philadelphia, PA, USA

W. Matthias · R. J. Thielen · D. Chin ·

C. D. Saffore $(\bowtie)$

Health Economics and Outcomes Research, AbbVie Inc., $1 \mathrm{~N}$ Waukegan Road, Dept. GMH1, ABV1 4NW102-04, North Chicago, IL 60064-6078, USA

e-mail: christopher.saffore@abbvie.com

W. Matthias · R. J. Thielen · D. Chin AbbVie Inc., North Chicago, IL, USA
}

(tsDMARDs) or visiting a rheumatologist. Outcomes were stratified by race/ethnicity, sex, and insurance coverage, with outcomes determined for commercial insurance, Medicare, and Medicaid enrollees. Differences observed in outcomes were numerical in nature.

Results: Prevalences of PsA and AS were highest for Medicare enrollees (320 and 156 per 100,000 persons $[0.32$ and $0.16 \%]$, respectively) and lowest for Medicaid enrollees (132 and 71 per $100,000$ persons [0.13 and $0.07 \%]$, respectively). White patients had the greatest prevalence versus patients of other races/ethnicities. Females had a higher prevalence of PsA than males, while AS prevalence was generally lower for females versus males for each insurance category. The percentage of patients prescribed bDMARDs/tsDMARDs was highest for commercial insurance enrollees (PsA 63\%, AS 43\%) and lowest for Medicare enrollees (PsA 21\%, AS $11 \%$ ). The proportion of patients who saw a rheumatologist was lower for Medicaid enrollees (PsA 12\%, AS 10\%) than for commercial insurance or Medicare enrollees (PsA 68\%, 55\%; AS $67 \%, 42 \%)$. For commercial insurance and Medicare enrollees, the percentage of patients visiting a rheumatologist was similar by race/ ethnicity but higher for females versus males.

Conclusions: The prevalence and treatment of PsA and AS differs by race/ethnicity, insurance coverage, and sex in the USA. Efforts for improving access to care are needed to improve outcomes among all patients. 
Keywords: Ankylosing spondylitis; Commercial insurance; Medicare; Medicaid; Prevalence; Psoriatic arthritis; Rheumatologist; Targeted immunomodulator; USA

\section{Key summary points}

Why carry out this study?

Patients in the USA with psoriatic arthritis (PsA) and ankylosing spondylitis (AS) experience delays in diagnosis and may receive inadequate treatment.

Disease prevalence and treatment patterns, stratified by race/ethnicity, sex, and insurance coverage in the USA, for PsA and AS have not been extensively evaluated.

A retrospective, cross-sectional US claims database analysis for 2019 was performed in patients with PsA and AS, evaluating prevalence and percentage of patients receiving biologic disease-modifying antirheumatic drugs (DMARDs) or targeted synthetic DMARDs, and the proportion visiting a rheumatologist.

\section{What was learned from this study?}

Disease prevalence and treatment for PsA and AS varies considerably among patients based on their race/ethnicity, sex, and insurance coverage.

Strategies for improving access to optimal care are needed for patients to obtain better outcomes.

\section{INTRODUCTION}

Psoriatic arthritis (PsA) and ankylosing spondylitis (AS) are members of the spondyloarthritis family of interrelated diseases that share various clinical manifestations, including inflammatory back pain, peripheral arthritis, enthesitis, and dactylitis, as well as extra-

articular features such as uveitis, psoriasis, and inflammatory bowel disease [1]. In the USA, the prevalence estimates for PsA and AS range from 0.06 to $0.25 \%$ and from 0.04 to $0.5 \%$, respectively [2-4]. While PsA is predominantly a peripheral disease, AS is primarily an axial disease $[1,5]$. However, $24 \%$ of patients who are diagnosed with PsA or AS can be classified for both diseases based on Classification of PsA (CASPAR) criteria or the modified New York (mNY) diagnostic criteria for AS [6].

Per clinical treatment guidelines for PsA and AS, biologic disease-modifying antirheumatic drugs (bDMARDs)/targeted synthetic DMARDs (tsDMARDs), such as tumor necrosis factor (TNF) inhibitors, are recommended as effective treatment options to manage and control these diseases [7, 8]. Additionally, conventional synthetic DMARDs, such as methotrexate and sulfasalazine, are indicated for use in PsA, although these medications are not indicated for use in patients with AS who do not have comorbid peripheral arthritis [7,8].

Despite published guidelines, patients with PsA and AS often receive inadequate care [9-11]. Patients with PsA encounter various treatment obstacles, including inadequate access to effective therapies and lack of treatment coordination between specialists and primary care providers [9]. For patients with AS in the USA, over $50 \%$ are prescribed opioids or corticosteroids by non-rheumatology providers, despite adverse events associated with these treatments [11].

Patients with PsA and AS also experience extensive diagnosis delays [9-11]. For patients with PsA, the disease often goes underdiagnosed, with diagnosis delays $>4$ years and with low referral frequencies to a rheumatologist $[9,12]$. With AS, diagnosis can be substantially delayed due to difficulty in discerning between inflammatory back pain versus mechanical back pain by primary care and non-rheumatologist health care providers [10, 11]. Additionally, slow-developing radiographic features may also impact delayed diagnosis for AS by up to 14 years [10]. Furthermore, only $37 \%$ of patients with AS are diagnosed by a rheumatologist, based on a retrospective analysis of administrative claims data from 2000 to 2012 [11]. 
Consequently, because patients with PsA and AS do not receive the proper, timely diagnosis and treatment, they experience undue symptom suffering, reduced quality of life, and irreversible outcomes, such as joint loss $[13,14]$.

One issue that has been inadequately evaluated are differences in diagnosis and treatment patterns in the USA by race/ethnicity, sex, and insurance coverage for patients with PsA or AS. To address this, we conducted a study of patients in the USA with commercial insurance; Medicare, which primarily insures individuals aged $\geq 65$ years; and Medicaid, which provides insurance for low-income individuals. Based on 2019 US census statistics, enrollment in commercial insurance/public insurance (the latter including Medicare and Medicaid) was 70/34\%, $55 / 42 \%, 74 / 25 \%$, and $52 / 36 \%$ for whites, blacks, Asians, and Hispanics, respectively [15]. In the present study, we assessed differences in the prevalence of PsA and AS, the use of bDMARDs/tsDMARDs, and rheumatologist visits based on patient race/ethnicity and sex.

\section{METHODS}

\section{Study Design and Population}

This was a retrospective, cross-sectional study using the Medicaid multi-state segment of the IBM ${ }^{\circledR}$ MarketScan ${ }^{\circledR}$ Commercial Claims and Encounters Supplemental Database and Optum Insight Clinformatics ${ }^{\circledR}$ Data Mart database from 1 January 2019 to 31 December 2019. The MarketScan Multi-State Medicaid Database contains claims data from approximately 36 million enrollees in 11 states [16]. This database includes inpatient, outpatient, pharmaceutical, and long-term care enrollment data, along with information on standard demographic variables and race [16]. The Optum database includes approximately 15-18 million patients from across the USA who were enrolled in commercial insurance or Medicare Advantage plans [17]. This database includes individual-level medical and pharmacy claims, laboratory results, member hospitalizations, and member eligibility data.
Patients aged $\geq 18$ years with $\geq 1$ International Classification of Diseases, Ninth Revision (ICD-9) (696.0) or ICD-10 (L40.5x) diagnosis codes for PsA and ICD-9 (720.0) or ICD-10 (M45.x) diagnosis codes for AS in 2019 and continuous medical and pharmacy enrollment during the calendar year were included in our analysis from both databases.

All database records were de-identified and made compliant with US patient confidentiality requirements, including the Health Insurance Portability and Accountability Act (HIPAA) of 1996 [16, 17]. Because of this, institutional review board approval was not required.

\section{Outcomes}

Outcomes evaluated were disease prevalence and prevalence of bDMARDs/tsDMARDs use and rheumatologist visits. Disease prevalence (per 100,000 persons) was estimated using the number of patients that met the PsA or AS definition described above as the numerator and the number of patients aged $\geq 18$ years with continuous enrollment (during the calendar year) as the denominator. bDMARD/tsDMARD utilization was estimated as the proportion of patients diagnosed with PsA or AS with $\geq 1$ claim for a bDMARD/tsDMARD during the calendar year. Prescriptions for bDMARDs/ tsDMARDs were identified using National Drug Codes and Healthcare Common Procedure Coding System codes. bDMARDs/tsDMARDs for PsA included abatacept, adalimumab, apremilast, certolizumab, etanercept, golimumab, infliximab, ixekizumab, secukinumab, tofacitinib, and ustekinumab. bDMARDs/tsDMARDs for AS included adalimumab, certolizumab, etanercept, golimumab, infliximab, ixekizumab, and secukinumab. The proportion of patients who visited a rheumatologist was estimated as the number of patients with $\geq 1$ visit with a rheumatologist during the calendar year divided by the number of patients diagnosed with PsA or AS.

Outcomes were stratified by patient-reported race/ethnicity (white, black, Asian, Hispanic, and unspecified), sex, and insurance coverage group (commercial, Medicare, Medicaid), with outcomes determined for commercial, 
Medicare, and Medicaid patients for 2019. For Medicaid patients, data for the Asian population and region were not available.

\section{Statistical Analyses}

Demographic and baseline characteristics (age, sex, region, and Charlson score) were summarized with descriptive statistics (mean and standard deviation [SD] for continuous endpoints, and number $(n)$ with percentage for categorical endpoints) for the overall population and for patients with PsA or AS separately. Categorical variables were reported as frequency and percentages. As this was a descriptive study, statistical analyses were not performed for comparing groups; therefore all differences observed in outcomes were numerical in nature. All data analyses were performed using the Instant Health Data platform (Panalgo, Boston, MA, USA).

\section{RESULTS}

\section{Study Population}

Of those who met the inclusion criteria, 14,896 , 16,469 , and 5785 patients with PsA had commercial insurance, Medicare, and Medicaid, respectively, in 2019 (Table 1). Among the patients with AS, 6664, 8019, and 3111 patients had commercial insurance, Medicare, and Medicaid, respectively (Table 2).

The mean age of patients with PsA or AS was 69 years for Medicare enrollees and 47-50 years for enrollees in the other insurance groups (Tables 1,2). By comparison, the mean age of the overall population was older (73 years) for Medicare enrollees (73 years) and younger for commercial insurance and Medicaid enrollees (42-45 years) (Electronic Supplementary Material [ESM] Table S1). The age of patients with AS or PsA was generally similar between the different races within their respective disease and insurance groups. The majority of patients in this study were white (57-74\%), irrespective of disease and insurance group, similar to the overall population in this study.
The proportion of patients in this study who were female was highest for Medicaid enrollees, accounting for $70 \%$ of patients with PsA (vs. $53 \%$ of those with commercial insurance and $60 \%$ of those with Medicare) and for $55 \%$ of patients with AS (vs. $48 \%$ of those with commercial insurance and $47 \%$ of those with Medicare) (Tables 1, 2). For the overall population in this study, the proportion of patients who were female was 64,49 , and $58 \%$ for Medicaid, commercial insurance, and Medicare enrollees, respectively (ESM Table S1). The highest proportion of females was found among black patients, while the lowest proportion was found among Asian patients for the given disease and insurance group.

In terms of region of the USA, $46 \%$ with commercial insurance were from the South for either disease (compared with $41 \%$ of the overall population) (Tables 1,2 and ESM Table S1). The highest proportion of Medicare patients with PsA and AS also came from the South (46-47\%, compared with $42 \%$ for the overall population). No regional data were available for Medicaid patients.

\section{Prevalence of PsA and AS}

Prevalence of PsA in the USA was highest among patients with Medicare (320 per 100,000 persons $[0.32 \%]$ ) and lowest among those with Medicaid (132 per 100,000 persons [0.13\%]), and it was 225 per 100,000 persons $(0.23 \%)$ for patients with commercial insurance (Fig. 1a). The prevalence of PsA was highest among white patients compared with the other race/ethnicity groups, irrespective of insurance group, with a prevalence of 273,340 , and 193 per 100,000 persons $(0.27,0.34$, and $0.19 \%)$ for those with commercial insurance, Medicare, and Medicaid, respectively. Prevalence of PsA among the other race/ethnicity groups was similar for commercial insurance enrollees (128-151 per 100,000 persons [0.13-0.15\%]); approximately 190 per 100,000 persons $(0.19 \%)$ for Medicare enrollees, with the exception of Hispanic patients (302 per 100,000 persons [0.30\%]); and 37 and 89 per 100,000 persons (0.04 and $0.09 \%$ ) for black and Hispanic patients with Medicaid, respectively. 
Table 1 Baseline characteristics for patients with psoriatic arthritis

\begin{tabular}{|c|c|c|c|c|c|c|}
\hline $\begin{array}{l}\text { Type of insurance coverage } \\
\text { Commercial }\end{array}$ & All & Asian & Black & White & Hispanic & Not specified \\
\hline$N$ & 14,896 & 421 & 669 & 10,351 & 1167 & 2288 \\
\hline Age (years), mean (SD) & $49.9(11.3)$ & $46.4(10.5)$ & $49.0(11.3)$ & $50.7(11.2)$ & $48.2(11.1)$ & $48.4(11.5)$ \\
\hline Female, $n(\%)$ & $7817(52.5)$ & $169(40.1)$ & $387(57.8)$ & $5432(52.5)$ & $633(54.2)$ & $1196(52.3)$ \\
\hline \multicolumn{7}{|l|}{ Region, $n(\%)^{\mathrm{a}}$} \\
\hline Midwest & $3926(26.4)$ & $73(17.3)$ & $111(16.6)$ & $3022(29.2)$ & $100(8.6)$ & $620(27.1)$ \\
\hline Northeast & $1637(11.0)$ & $51(12.1)$ & $34(5.1)$ & $1188(11.5)$ & $85(7.3)$ & $279(12.2)$ \\
\hline South & $6846(46.0)$ & $155(36.8)$ & $486(72.6)$ & $4515(43.6)$ & $676(57.9)$ & $1014(44.3)$ \\
\hline West & $2461(16.5)$ & $142(33.7)$ & $37(5.5)$ & $1613(15.6)$ & $299(25.6)$ & $370(16.2)$ \\
\hline Charlson score, mean (SD) & $0.79(1.29)$ & $0.56(0.98)$ & $0.86(1.33)$ & $0.80(1.31)$ & $0.85(1.29)$ & $0.72(1.21)$ \\
\hline \multicolumn{7}{|l|}{ Medicare } \\
\hline$N$ & 16,469 & 273 & 867 & 9540 & 1398 & 4391 \\
\hline Age (years), mean (SD) & $68.7(9.0)$ & $70.4(7.9)$ & $68.0(9.8)$ & $69.5(8.8)$ & $69.2(10.0)$ & $67.0(8.8)$ \\
\hline Female, $n(\%)$ & $9941(60.4)$ & $147(53.8)$ & $608(70.1)$ & $5673(59.5)$ & $887(63.4)$ & $2626(59.8)$ \\
\hline \multicolumn{7}{|l|}{ Region, $n(\%)^{a}$} \\
\hline Midwest & $2885(17.5)$ & $33(12.1)$ & $121(14.0)$ & $1960(20.5)$ & $61(4.4)$ & $710(16.2)$ \\
\hline Northeast & $2404(14.6)$ & $42(15.4)$ & $57(6.6)$ & $1585(16.6)$ & $144(10.3)$ & $576(13.1)$ \\
\hline South & $7753(47.1)$ & $82(30.0)$ & $643(74.2)$ & $4052(42.5)$ & $858(61.4)$ & $2118(48.2)$ \\
\hline West & $3429(20.8)$ & $116(42.5)$ & $46(5.3)$ & $1944(20.4)$ & $336(24.0)$ & $987(22.5)$ \\
\hline Charlson score, mean (SD) & $1.88(2.0)$ & $1.89(1.98)$ & $2.16(2.06)$ & $1.88(2.01)$ & $2.15(2.11)$ & $1.76(1.94)$ \\
\hline \multicolumn{7}{|l|}{ Medicaid } \\
\hline$N$ & 5785 & & 515 & 4295 & 199 & 776 \\
\hline Age (years), mean (SD) & $49.5(13.2)$ & & $52.3(13.4)$ & $49.3(13.2)$ & $49.4(12.8)$ & $48.9(13.2)$ \\
\hline Female, $n(\%)$ & $4024(69.6)$ & & $402(78.1)$ & $3034(70.6)$ & $128(64.3)$ & $460(59.3)$ \\
\hline Charlson score, mean (SD) & $1.52(1.79)$ & & $1.75(1.97)$ & $1.55(1.80)$ & $1.30(1.72)$ & $1.29(1.61)$ \\
\hline
\end{tabular}

$N A$ not available, $S D$ standard deviation

${ }^{a}$ Percentage of those with available regional data

When stratified by sex, the prevalence of PsA was higher among females than males for those enrolled in commercial insurance, Medicare, and Medicaid (242 vs. 208, 336 vs. 299, and 143 vs. 111 per 100,000 persons [0.24 vs. $0.21 \%$, 0.34 vs. $0.30 \%, 0.14$ vs. $0.11 \%]$, respectively) (ESM Fig. S1A). This trend was also observed within the racial/ethnic groups, with the exception of Asians, where prevalence of PsA was lower among females than males for those who had commercial insurance (109 vs. 144 persons per 100,000 persons [0.11 vs. $0.14 \%]$ ) and similar for those with Medicare (ESM Fig. S1A). 
Table 2 Baseline characteristics for patients with ankylosing spondylitis

\begin{tabular}{|c|c|c|c|c|c|c|}
\hline $\begin{array}{l}\text { Type of insurance coverage } \\
\text { Commercial }\end{array}$ & All & Asian & Black & White & Hispanic & Not specified \\
\hline$N$ & 6664 & 245 & 388 & 4460 & 589 & 982 \\
\hline Age (years), mean (SD) & $46.8(12.5)$ & $43.6(11.7)$ & $48.1(12.4)$ & $47.6(12.5)$ & $44.9(12.2)$ & $45.0(12.3)$ \\
\hline Female, $n(\%)$ & $3206(48.1)$ & $93(38.0)$ & $204(52.6)$ & $2113(47.4)$ & $310(52.6)$ & $486(49.5)$ \\
\hline \multicolumn{7}{|l|}{ Region, $n(\%)^{\mathrm{a}}$} \\
\hline Midwest & $1517(22.8)$ & $37(15.1)$ & $56(14.4)$ & $1156(25.9)$ & $57(9.7)$ & $211(21.5)$ \\
\hline Northeast & $570(8.6)$ & $31(12.7)$ & $27(7.0)$ & $375(8.4)$ & $40(6.8)$ & $97(9.9)$ \\
\hline South & $3077(46.2)$ & $77(31.4)$ & $278(71.6)$ & $1955(43.8)$ & $316(53.7)$ & $451(45.9)$ \\
\hline West & $1477(22.2)$ & $97(39.6)$ & $25(6.4)$ & $968(21.7)$ & $171(29.0)$ & $216(22.0)$ \\
\hline Charlson score, mean (SD) & $0.80(1.28)$ & $0.67(1.12)$ & $1(1.38)$ & $0.80(1.30)$ & $0.90(1.30)$ & $0.71(1.15)$ \\
\hline \multicolumn{7}{|l|}{ Medicare } \\
\hline$N$ & 8019 & 156 & 531 & 4542 & 749 & 2,041 \\
\hline Age (years), mean (SD) & $69.1(10.2)$ & $71.2(8.9)$ & $67.3(10.4)$ & $70.3(10.0)$ & $69.5(10.3)$ & $66.8(10.3)$ \\
\hline Female, $n(\%)$ & $3740(46.6)$ & $67(42.9)$ & $299(56.3)$ & $2008(44.2)$ & $399(53.3)$ & $967(47.4)$ \\
\hline \multicolumn{7}{|l|}{ Region, $n(\%)^{\mathrm{a}}$} \\
\hline Midwest & $1298(16.2)$ & $3(1.9)$ & $63(11.9)$ & $879(19.4)$ & $26(3.5)$ & $327(16.0)$ \\
\hline Northeast & $937(11.7)$ & $38(24.4)$ & $28(5.3)$ & $601(13.2)$ & $60(8.0)$ & $210(10.3)$ \\
\hline South & $3681(45.9)$ & $45(28.8)$ & $407(76.6)$ & $1814(39.9)$ & $434(57.9)$ & $981(48.1)$ \\
\hline West & $2104(26.2)$ & $70(44.9)$ & $34(6.4)$ & $1249(27.5)$ & $229(30.6)$ & $522(25.6)$ \\
\hline Charlson score, mean (SD) & $2.06(2.08)$ & $1.97(1.90)$ & $2.24(2.15)$ & $2.06(2.08)$ & $2.11(2.00)$ & $2.00(2.09)$ \\
\hline \multicolumn{7}{|l|}{ Medicaid } \\
\hline$N$ & 3111 & & 573 & 1955 & 119 & 464 \\
\hline Age (years), mean $(\mathrm{SD})$ & $48.8(13.9)$ & & $50.9(14.1)$ & $48.2(13.6)$ & $48.1(15.1)$ & $49.0(14.7)$ \\
\hline Female, $n(\%)$ & $1703(54.7)$ & & $337(58.8)$ & $1093(55.9)$ & $60(50.4)$ & $213(45.9)$ \\
\hline Charlson score, mean (SD) & $1.42(1.74)$ & & $1.63(1.86)$ & $1.40(1.73)$ & $1.25(1.67)$ & $1.32(1.60)$ \\
\hline
\end{tabular}

$N A$ not available, $S D$ standard deviation

${ }^{a}$ Percentage of those with available regional data

Prevalence of AS was highest among patients with Medicare (156 per 100,000 persons [0.16\%]), lowest among patients with Medicaid (71 per 100,000 persons [0.07\%]), and 101 per 100,000 persons [0.10\%] among those with commercial insurance (Fig. 1B). The prevalence of AS was higher among white patients compared with other race/ethnicity groups for those with commercial insurance (118 per 100,000 persons [0.12\%]) and Medicaid (88 per 100,000 persons [0.09\%]), whereas among Medicare enrollees both white and Hispanic patients had the highest prevalence of AS (162 per 100,000 persons [0.16\%]). AS prevalence 
A

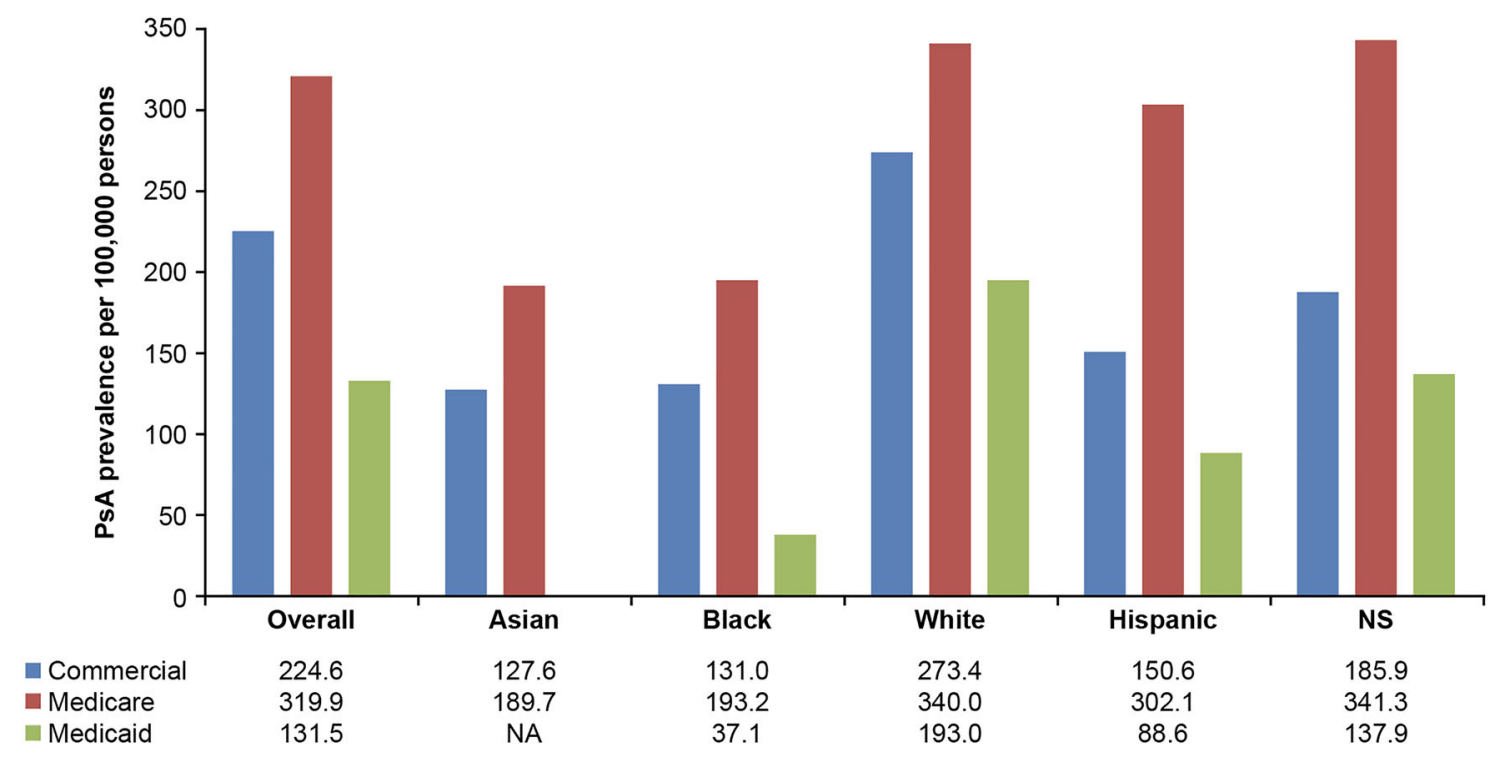

B

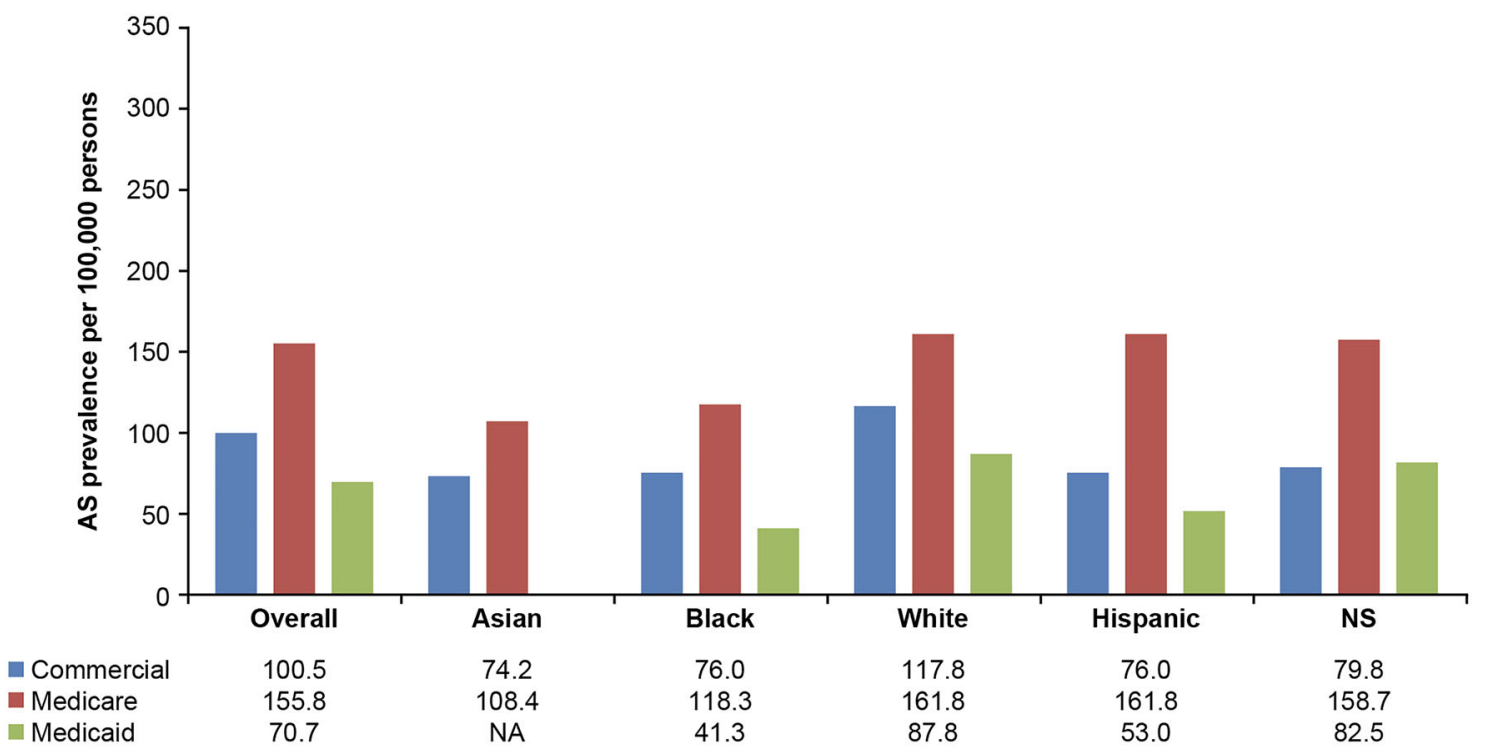

Fig. 1 Prevalence of PsA and AS in the USA by race and insurance coverage. a Prevalence of PsA, b prevalence of AS. $A S$ Ankylosing spondylitis, $N A$ not available, $N S$ not specified, $P s A$ psoriatic arthritis

was similar between the other race/ethnicity groups with commercial insurance (74-76 per 100,000 persons [0.07\%-0.08\%]), Medicare (108 and 119 per 100,000 persons [0.11\% and $0.12 \%$ ] for Asian and black patients, respectively), and
Medicaid (41 and 53 per 100,000 persons [0.04\% and 0.05\%] for black and Hispanic patients, respectively).

When stratified by sex, the prevalence of AS was lower for females than males among those 
enrolled in Medicare and Medicaid (126 vs. 196 and 61 vs. 89 per 100,000 persons [0.13 vs. $0.20 \%$ and 0.06 vs. $0.09 \%]$, respectively) and similar for commercial insurance enrollees (99-102 per 100,000 persons [0.1\%]; ESM Fig. S1B). Similar trends for males and females were noted for the individual race and ethnicity groups compared with all Medicare and Medicaid patients with AS. For those with commercial insurance, prevalence of AS among females versus males was lower for Asian patients, higher for Hispanic patients, and similar for black and white patients.

\section{Prevalence of bDMARD/tsDMARD Utilization}

The percentage of patients with PsA who were prescribed bDMARDs/tsDMARDs in the USA was greater for commercial insurance (63\%) enrollees than for Medicare (21\%) or Medicaid (37\%) enrollees (Fig. 2a). Similar percentages of commercially insured patients with PsA were prescribed bDMARDs/tsDMARDs among the different race/ethnicity groups (60-64\%), whereas, among Medicare enrollees the percentage ranged from $20 \%$ for white patients to $29 \%$ for black patients and for Medicaid enrollees it ranged from $29 \%$ for black patients to $45 \%$ for Hispanic patients.

A lower percentage of female than male patients with PsA who had commercial insurance were prescribed bDMARDs/tsDMARDs (60 vs. $66 \%$ ), with similar differences noted for the respective race/ethnicity groups (ESM Fig. S2A). For patients with Medicare, similar percentages of female and male patients were prescribed bDMARDs/tsDMARDs (20 and 22\%, respectively), while for patients with Medicaid, a slightly lower percentage of females were prescribed bDMARDs/tsDMARDs (36 vs 39\%; ESM Fig. S2A). Similar findings were generally observed with Medicare and Medicaid enrollees for the individual race/ethnicity groups.

For patients with AS, the percentage of patients prescribed bDMARDs/tsDMARDs was considerably higher for those with commercial insurance $(43 \%)$ compared with those with Medicare (11\%) or Medicaid (20\%) (Fig. 2B).
The percentage of patients who were prescribed bDMARDs/tsDMARDs ranged from 38\% for Hispanic patients to $45 \%$ for Asian patients for those with commercial insurance, $8 \%$ for Asian patients to $14 \%$ for black patients for those on Medicare, and $13 \%$ for black patients to $24 \%$ for Hispanic patients for those on Medicaid.

Similar differences between female and male patients with AS in the percentages receiving bDMARDs/tsDMARDs were noted as observed for patients with PsA. The percentage of female versus male patients with AS receiving bDMARDs/tsDMARDs was lower for those with commercial insurance (38 vs. $47 \%$ ), similar for Medicare enrollees (11\%), and slightly lower for Medicaid enrollees (18 vs. 21\%) (ESM Fig. S2B). For the different race/ethnicity groups, the percentage of female versus male patients receiving bDMARDs/tsDMARDs was generally similar among all patients for the respective insurance groups, with the exception of Hispanic Medicaid enrollees, where a higher percentage of females versus males received bDMARDs/tsDMARDs.

\section{Prevalence of Visits to Rheumatologist}

The percentage of patients with PsA who visited a rheumatologist was much lower for those with Medicaid (12\%) compared with those who had commercial insurance $(68 \%)$ or Medicare $(55 \%)$ (Fig. 3a). For patients with commercial insurance or Medicaid, the proportion of patients with PsA visiting a rheumatologist were similar among race/ethnicity groups (64-69\% and $10-11 \%$, respectively), while for patients with Medicare, the percentages were slightly higher for black and white patients (55-56\%) compared with Asian and Hispanic patients (47-52\%).

For female patients with PsA and commercial insurance, an overall higher percentage, compared with males, visited a rheumatologist, with the same trend seen among white and Hispanic patients (ESM Fig. S3A). With Medicare enrollees, a higher percentage of females compared with males visited a rheumatologist, for all patients and for each race/ethnicity group, 
A

PSA

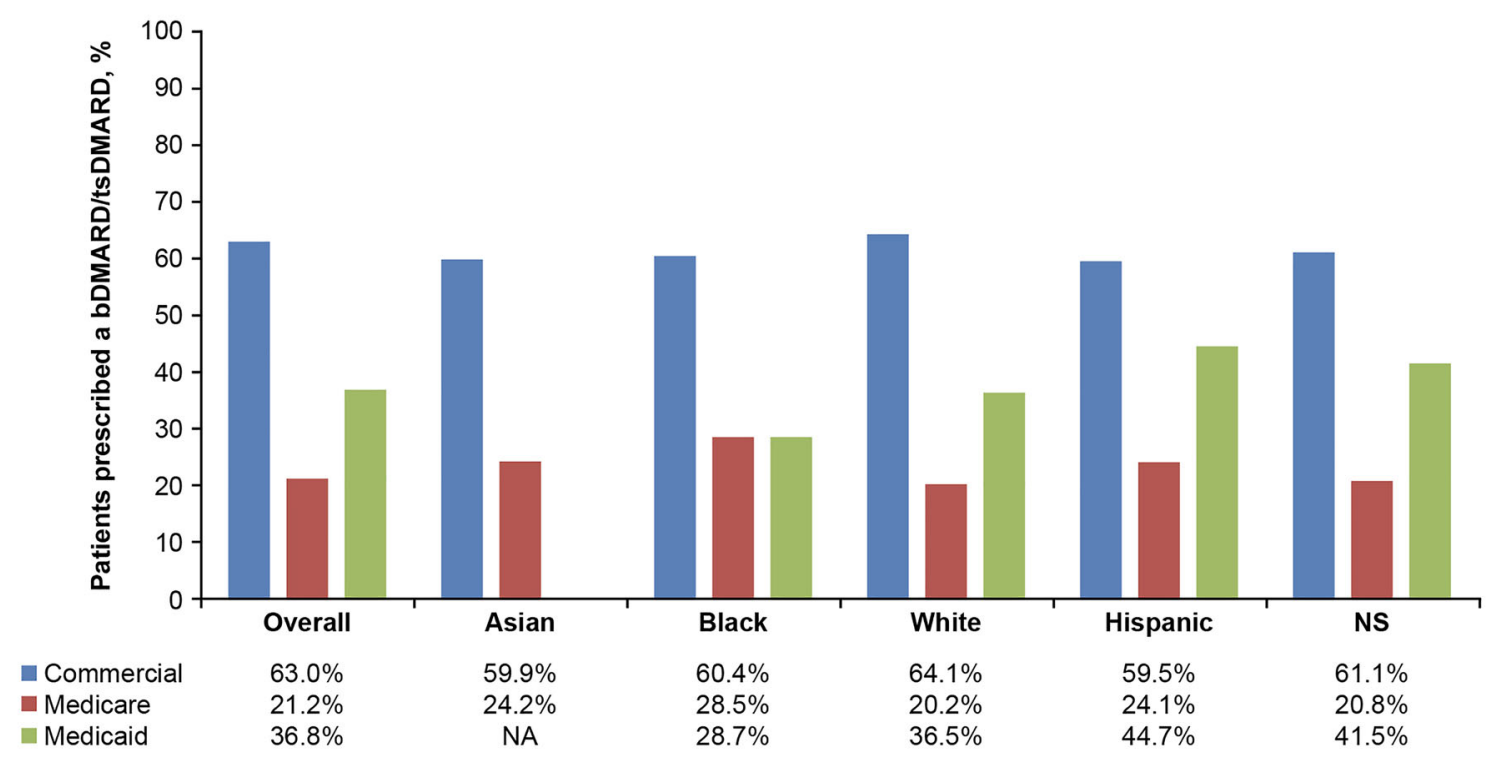

B

AS

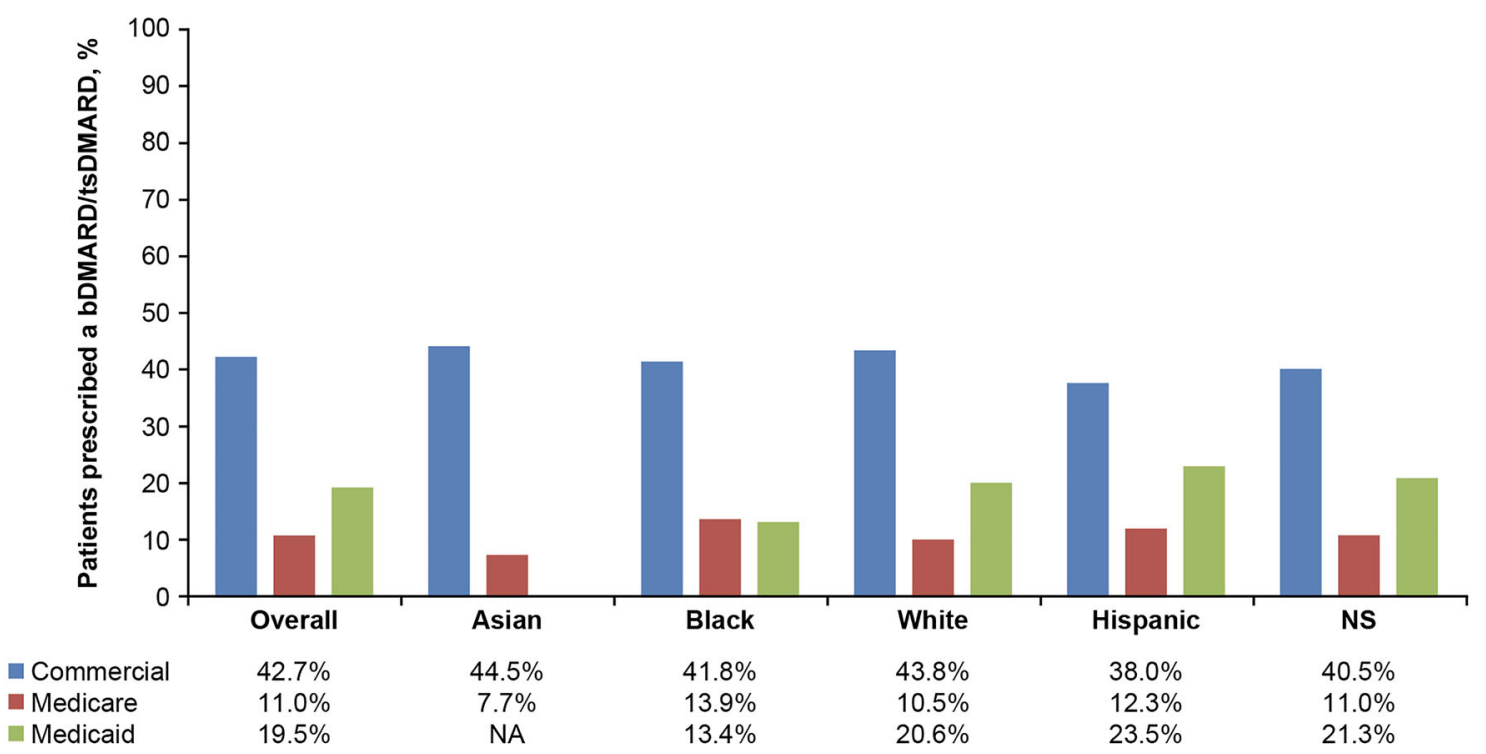

Fig. 2 bDMARD/tsDMARD utilization among patients with PsA and AS in the USA by race and insurance coverage. a Percentage of patients with PsA prescribed a bDMARD/tsDMARD, b percentage of patients with AS prescribed a bDMARD/tsDMARD. $A S$ ankylosing

while the percentages were similar with those for Medicaid enrollees.

spondylitis, $b D M A R D$ biologic DMARD, DMARD disease-modifying antirheumatic drug, $N A$ not available, $N S$ not specified, $P_{S} A$ psoriatic arthritis, tsDMARD targeted synthetic DMARD

For patients with AS, a considerably lower percentage of Medicaid enrollees visited a rheumatologist (10\%) compared to those who 
A

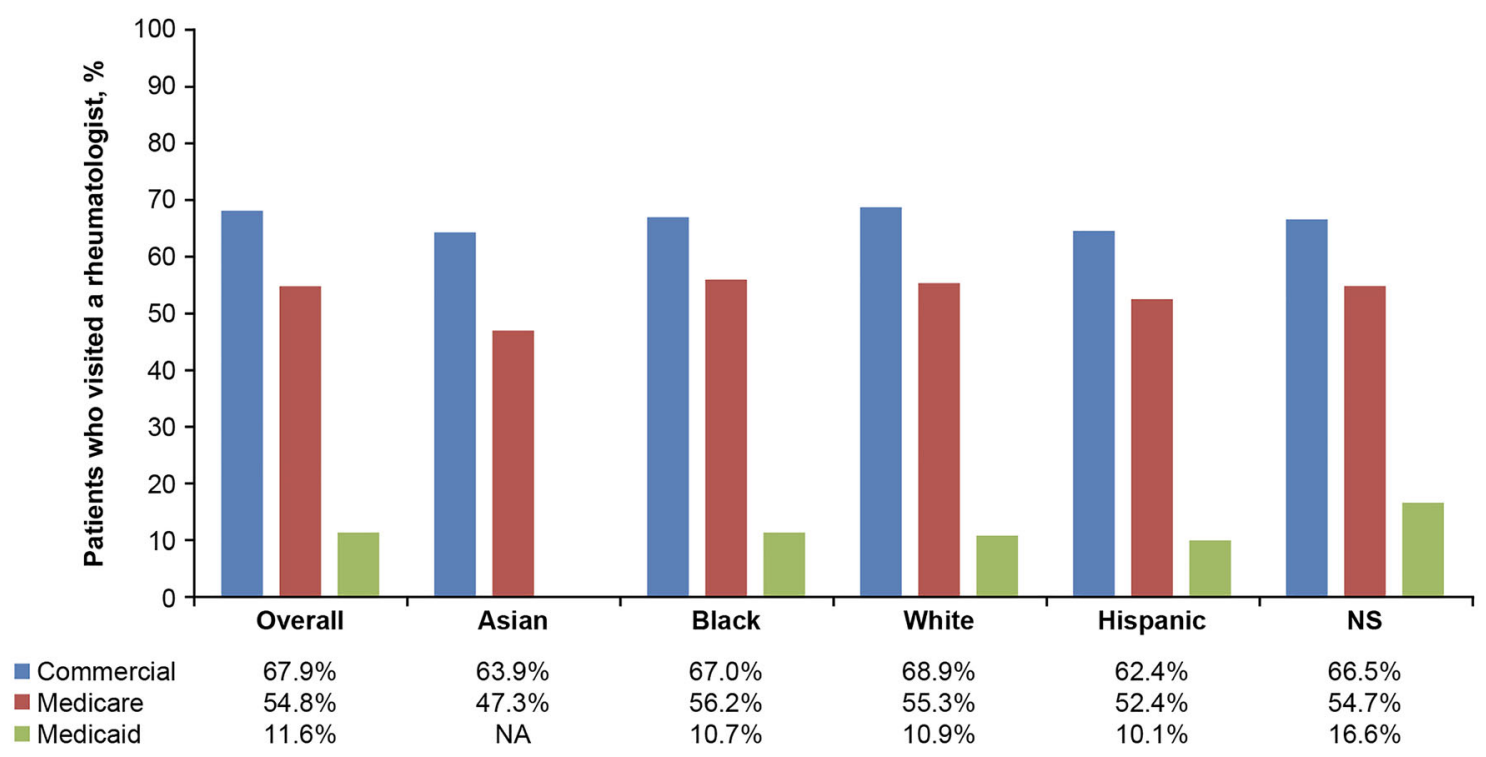

B

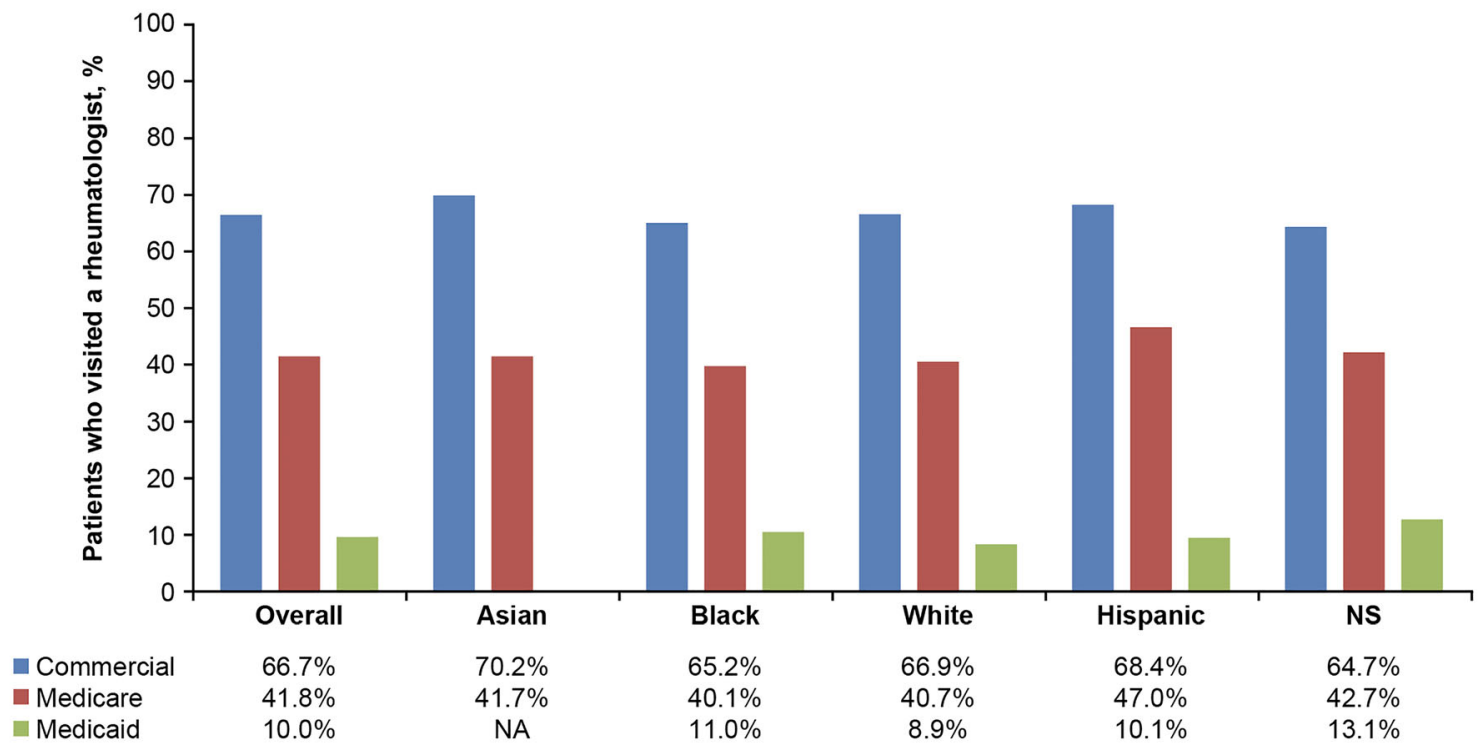

Fig. 3 Visits to the rheumatologist among patients with PsA and AS in the USA by race and insurance coverage. a Percentage of patients with PsA visiting a

had commercial insurance $(67 \%)$ or Medicare (42\%), comparable to that observed for patients with PsA (Fig. 3B). Similar percentages of patients with AS from the different race/ rheumatologist, b Percentage of patients with AS visiting a rheumatologist. $A S$ ankylosing spondylitis, $N A$ not available, $N S$ not specified, $P_{S} A$ psoriatic arthritis

ethnicity groups visited a rheumatologist among those with commercial insurance $(65-70 \%)$ or Medicare $(40-42 \%)$, with the exception of Hispanic Medicare enrollees (47\%), 
while percentages ranged from 9 to $11 \%$ for Medicaid enrollees.

Overall, a higher percentage of female versus male patients with AS visited a rheumatologist among those with commercial insurance (70 vs. $64 \%$ ), Medicare (50 vs. $35 \%$ ), or Medicaid (11 vs. 9\%) (ESM Fig. S3B). For the different race/ethnicity groups, a higher percentage of females versus males with commercial insurance visited a rheumatologist (except for black patients), Medicare, and Medicaid (except for black and Hispanic patients).

\section{DISCUSSION}

In this large, descriptive, retrospective, crosssectional US claims database analysis, the prevalence of PsA and AS was found to vary greatly based on insurance coverage, ranging per 100,000 persons from 132 to 320 (0.13-0.32\%) for patients with PsA and 71-156 $(0.07-0.16 \%)$ for patients with AS. Although variable, these results are consistent with previously reported estimates [2-4].

We also observed that the prevalence of PsA and AS was highest among white patients compared with other racial/ethnic groups, irrespective of insurance coverage, with differences as high as 5.2-fold between black and white Medicaid patients with PsA. Few studies have reported on racial and ethnic differences in prevalence of PsA and AS [18-20]. For PsA, differences in the prevalence between white and black patients were previously estimated as approximately twofold, consistent with the findings for the commercial insurance and Medicare populations in this study $[18,19]$. For AS, prevalence differed by as much as tenfold between white and black patients in a retrospective clinical database study of patients between 1999 and 2017, while in the current study it was at most twofold [20]. Various factors may be involved in this prevalence difference between white and black patients. HLAB27, a molecule associated with AS based on genetic analyses, is 7 times more frequently expressed in whites than blacks [21, 22]. As a consequence, physicians may not consider AS in their diagnosis of black patients, particularly those who are HLA-B27-negative [20, 23]. Access to care and advanced diagnostic techniques, such as magnetic resonance imaging for non-radiographic AS, are less available to black than white patients [23, 24]. Furthermore, for PsA, as psoriasis is more difficult to recognize in patients with darker skin phenotypes [25], these patients may be diagnosed with rheumatoid arthritis.

Prevalence of PsA was found to be higher among females than among males, irrespective of insurance coverage and, in general, race/ ethnicity. In a previous meta-analysis of 36 observational and clinical studies worldwide, prevalence was slightly higher for women than men ( 24.0 vs. $23.3 \%)$ [26]. The higher prevalence of PsA in women versus men observed in this study may be due to differences in behavior (i.e., women tend to go to the physician more than men [27]), study designs, time period evaluated, and/or the study population. For AS, our observation of females having a lower prevalence than males is consistent with findings reported previously [4].

While prevalence differed by race/ethnicity, treatment was similar among race/ethnicity subgroups in this study. The percentages of patients who were prescribed bDMARDs/ tsDMARDs were generally similar between the different racial/ethnic groups for those with commercial insurance or Medicare, while it was lower for black versus white patients who enrolled in Medicaid.

The overall percentage of patients with PsA and AS who received bDMARDs/tsDMARDs was at most 63 and 43\%, respectively, with the highest percentages being for those with commercial insurance. These values are similar to previous real-world claims reports which did not stratify by insurance group [4, 28]. Nevertheless, bDMARD/tsDMARD use for PsA and AS is low, particularly for patients enrolled in Medicare and, to a certain extent, those enrolled in Medicaid, indicating that there is still an unmet treatment need for these patient population populations. Furthermore, bDMARD/ tsDMARD use in Medicare patients may be underestimated since this study evaluated patients with the Medicare Advantage program [29], which represents one-third of all Medicare 
patients and does not include those who just have Medicare part A and B. Also, as Medicaid coverage varies by state, bDMARD/tsDMARD use could be lower for those states with restricted access to these medications.

For the different racial/ethnic groups with commercial insurance or Medicare, similar percentages of patients visited rheumatologists for both diseases. However, the overall percentage of patients visiting a rheumatologist was low, particularly for patients with Medicaid (approx. $10 \%)$. Such low rates can have a major impact on receiving proper care and obtaining disease control.

To the best of our knowledge, this is the first report to describe the association of insurance coverage with PsA and AS prevalence and treatment. We identified major differences in PsA and AS prevalence and treatment based on insurance coverage, particularly for Medicaid enrollees, among whom prevalence was substantially lower compared with commercial insurance enrollees with PsA and AS, respectively. Additionally, the percentage of patients with either PsA or AS who visited a rheumatologist or were prescribed bDMARDs/tsDMARDs were markedly lower for Medicaid versus commercial insurance enrollees. While it remains unclear why the prevalence is lower among Medicaid and Medicare enrollees, one possibility is that patients have less access to specialty care.

Previous reports have described insurancebased discrimination in health care for patients receiving public insurance, such as Medicaid, versus those with commercial insurance $[30,31]$. This has resulted in patients being unable to or having greater difficulty in receiving specialist care, prescription drugs, or routine medical care [30, 31]. The results presented in this study emphasize the need to provide better treatment and care for patients with PsA and AS who have government-funded insurance.

With respect to race/ethnicity, limited information has been published on its influence on PsA and AS prevalence and treatment, although racial disparities in treatment have been well documented for other diseases, including cardiovascular disease, cancer, and COVID-19 [32-34]. In previous studies, black patients with PsA or AS were found to have more severe disease than white patients, based on various disease and quality of life measurement criteria, including patient-physician global scores, PSORIasis Index of Quality of Life, and Short Form-36 mental component score for PsA and Bath AS Disease Activity Index, Bath AS Functional Index, Bath AS Radiographic Index, and the modified Stoke AS Spine score for AS $[19,20,35]$. Furthermore, studies have identified a greater prevalence of comorbidities among black patients compared to white patents with PsA or AS $[19,20]$. However, treatment rates with more effective therapies, such as bDMARDs/tsDMARDs, are lower by as much as 3.5-fold for patients with PsA and 1.6fold for patients with AS [19, 35]. While the current study did not find major differences between racial/ethnic groups in bDMARD/ tsDMARD utilization or in the prevalence of rheumatologist visits, previous studies have found gaps between groups $[19,20,35]$. This discrepancy may have been due to differences in study design, such as insurance coverage stratification.

Lower percentages of female versus male patients were prescribed bDMARDs/tsDMARDs among those who had commercial insurance, consistent with results reported previously for patients with AS who were not stratified by insurance group [4]. A variety of reasons for differences in treatment based on sex have been reported earlier, which could potentially be factors in this current study, including physician and patient behaviors and variances in disease manifestations and treatment response [36]. However, because of limitations imposed by this being a claims database analysis, such factors could not be evaluated in the current study.

The use of two large US databases that contained commercial, Medicare, and Medicaid deidentified beneficiary information provides a strong basis for evaluating prevalence and treatment differences based on race/ethnicity, insurance coverage, and sex. However, there are limitations in using this approach. As this was a descriptive study, statistical analyses were not performed to compare groups. Future studies are needed to further understand the influence 
and associations of race/ethnicity and sex on these outcomes by insurance type. As data for only Medicare Advantage patients, which represent approximately one-third of Medicare patients [29], were available, the results are not generalizable to the entire Medicare population. Uninsured patients were not reported in this study, which could lead to an underestimation of prevalence. Consequently, this study is only generalizable to the types of insured patients represented in the MarketScan Multi-State Medicaid and Optum databases. It is possible that misclassifications in the insurance databases may occur and that this could lead to over- or underestimation of PsA and AS prevalence. Lastly, because different databases had to be used for the patient population enrolled in Medicaid, there is the potential for some reporting differences between the groups owing to database methodology. Additionally, information specifically on Asian patients and regional data were not available through the MarketScan Multi-State Medicaid Database, although it was available through the Optum database.

\section{CONCLUSIONS}

In summary, the prevalence of PsA and AS among patients in the USA was found to differ by racial/ethnic group, particularly between black and white patients, in this claims database analysis. Rheumatologic care and treatment of patients with PsA and AS were found to differ by type of insurance coverage. Patients with Medicaid had the lowest use of appropriate treatments and patients with commercial insurance had the highest use. Additionally, sex-related differences in treatment was found to exist for patients with PsA and AS, with a lower percentage of women being treated with bDMARDs/tsDMARDs for their disease. By characterizing patient populations with the greatest need for improvements in the care and treatment of PsA and AS, more emphasis can be directed by physicians, payers, and government to identify and remedy deficiencies in the health care system.

\section{ACKNOWLEDGEMENTS}

Funding. This work was supported by AbbVie Inc. AbbVie sponsored the study; contributed to the design; participated in collection, analysis, and interpretation of data, and in the writing, reviewing, and approval of the final version; and funded the Rapid Service fee and Open Access fee. No honoraria or payments were made for authorship.

Authorship. All named authors meet the International Committee of Medical Journal Editors (ICMJE) criteria for authorship for this article, take responsibility for the integrity of the work as a whole, and have given their approval for this version to be published.

Author Contributions. All authors contributed to the study conception and design, material preparation, data collection, and analysis. All authors were involved in the preparation and critical review of the manuscript and read and approved the final manuscript.

Medical Writing, Editorial, and Other Assistance. Medical writing assistance was provided by Alan Saltzman, PhD, CMPP, of Fishawack Facilitate Ltd, part of Fishawack Health, Conshohocken, PA, and was funded by AbbVie Inc., North Chicago, IL.

Disclosures. Alexis Ogdie has received consulting fees from Amgen, AbbVie, BMS, Celgene, CorEvitas (previously Corrona, LLC), Gilead, Janssen, Lilly, Novartis, Pfizer, and UCB; and has received grant support from the National Institutes of Health/National Institute of Arthritis and Musculoskeletal and Skin Diseases, Rheumatology Research Foundation, National Psoriasis Foundation, Pfizer, and Novartis. Wesley Matthias, Richard J. Thielen, Daniel Chin, and Christopher D. Saffore are employees of AbbVie and own AbbVie stock.

Compliance with Ethics Guidelines. All database records were de-identified and compliant with US patient confidentiality requirements, including the Health Insurance 
Portability and Accountability Act of 1996. Because of this, institutional review board approval was not required.

Data Availability. The datasets generated and/or analyzed during the current study are available from the corresponding author on reasonable request.

Open Access. This article is licensed under a Creative Commons Attribution-NonCommercial 4.0 International License, which permits any non-commercial use, sharing, adaptation, distribution and reproduction in any medium or format, as long as you give appropriate credit to the original author(s) and the source, provide a link to the Creative Commons licence, and indicate if changes were made. The images or other third party material in this article are included in the article's Creative Commons licence, unless indicated otherwise in a credit line to the material. If material is not included in the article's Creative Commons licence and your intended use is not permitted by statutory regulation or exceeds the permitted use, you will need to obtain permission directly from the copyright holder. To view a copy of this licence, visit http://creativecommons.org/licenses/by$\mathrm{nc} / 4.0 /$.

\section{REFERENCES}

1. Feld J, Chandran V, Haroon N, Inman R, Gladman D. Axial disease in psoriatic arthritis and ankylosing spondylitis: a critical comparison. Nat Rev Rheumatol. 2018;14:363-71.

2. Ogdie A, Weiss P. The epidemiology of psoriatic arthritis. Rheum Dis Clin North Am. 2015;41: 545-68.

3. Reveille JD. Epidemiology of spondyloarthritis in North America. Am J Med Sci. 2011;341:284-6.

4. Walsh J, Hunter T, Schroeder K, Sandoval D, Bolce R. Trends in diagnostic prevalence and treatment patterns of male and female ankylosing spondylitis patients in the United States, 2006-2016. BMC Rheumatol. 2019;3:39.

5. Feld J, Ye JY, Chandran V, et al. Is axial psoriatic arthritis distinct from ankylosing spondylitis with and without concomitant psoriasis? Rheumatology (Oxford). 2020;59:1340-6.

6. Jadon DR, Sengupta R, Nightingale A, et al. Axial Disease in Psoriatic Arthritis study: defining the clinical and radiographic phenotype of psoriatic spondyloarthritis. Ann Rheum Dis. 2017;76:701-7.

7. Ogdie A, Coates LC, Gladman DD. Treatment guidelines in psoriatic arthritis. Rheumatology (Oxford). 2020;59:i37-46.

8. Ward MM, Deodhar A, Gensler LS, et al. 2019 Update of the American College of Rheumatology/ Spondylitis Association of America/Spondyloarthritis Research and Treatment Network recommendations for the treatment of ankylosing spondylitis and nonradiographic axial spondyloarthritis. Arthritis Care Res (Hoboken). 2019;71: 1285-99.

9. Favier G, Gladman DD, Merola JF, Armstrong AW, Boehncke WH, Helliwell PS. Benchmarking care in psoriatic arthritis-the QUANTUM Report: a report from the GRAPPA 2016 Annual Meeting. J Rheumatol. 2017;44:674-8.

10. Deodhar A, Mease PJ, Reveille JD, et al. Frequency of axial spondyloarthritis diagnosis among patients seen by US Rheumatologists for Evaluation of Chronic Back Pain. Arthritis Rheumatol. 2016;68: 1669-76.

11. Deodhar A, Mittal M, Reilly P, et al. Ankylosing spondylitis diagnosis in US patients with back pain: identifying providers involved and factors associated with rheumatology referral delay. Clin Rheumatol. 2016;35:1769-76.

12. Mease PJ, Liu C, Siegel E, et al. Impact of clinical specialty setting and geographic regions on disease management in patients with psoriatic arthritis in the United States: a multicenter observational study. Am J Clin Dermatol. 2019;20:873-80.

13. Kavanaugh A, Helliwell P, Ritchlin CT. Psoriatic arthritis and burden of disease: patient perspectives from the population-based multinational assessment of psoriasis and psoriatic arthritis (MAPP) survey. Rheumatol Ther. 2016;3:91-102.

14. Law L, Beckman Rehnman J, Deminger A, Klingberg E, Jacobsson LTH, Forsblad-d'Elia H. Factors related to health-related quality of life in ankylosing spondylitis, overall and stratified by sex. Arthritis Res Ther. 2018;20:284.

15. Keisler-Starkey K, Bunch LN. Health Insurance coverage in the United States: 2019. 2020. https:// www.census.gov/content/dam/Census/library/ publications/2020/demo/p60-271.pdf. Accessed 9 Aug 2021. 
16. IBM Watson Health. Data brochure. IBM MarketScan Research Databases for life sciences researchers. $2020 . \quad$ https://www.ibm.com/downloads/cas/ OWZWJ0QO. Accessed 26 Feb 2021.

17. Optum. Clinformatics ${ }^{\circledR}$ data mart. 2017. https:// www.optum.com/content/dam/optum3/optum/ en/resources/white-papers/clinformatics-data-mart. pdf. Accessed 26 Feb 2021.

18. Gelfand JM, Stern RS, Nijsten T, et al. The prevalence of psoriasis in African Americans: results from a population-based study. J Am Acad Dermatol. 2005;52:23-6.

19. Kerr GS, Qaiyumi S, Richards J, et al. Psoriasis and psoriatic arthritis in African-American patientsthe need to measure disease burden. Clin Rheumatol. 2015;34:1753-9.

20. Singh DK, Magrey M. Racial differences in clinical features and comorbidities in ankylosing spondylitis in the United States. J Rheumatol. 2020;47: 835-8.

21. Reveille JD, Hirsch R, Dillon CF, Carroll MD, Weisman MH. The prevalence of HLA-B27 in the US: data from the US National Health and Nutrition Examination Survey, 2009. Arthritis Rheum. 2012;64:1407-11.

22. Chen B, Li J, He C, et al. Role of HLA-B27 in the pathogenesis of ankylosing spondylitis (review). Mol Med Rep. 2017;15:1943-51.

23. Karmacharya P, Balls-Berry JE, Davis JM 3rd. True difference or detection bias: racial differences in clinical features and comorbidities in Ankylosing Spondylitis in the United States. J Rheumatol. 2020;47:1150.

24. Obermeyer Z, Powers B, Vogeli C, Mullainathan S. Dissecting racial bias in an algorithm used to manage the health of populations. Science. 2019;366:447-53.

25. Alexis AF, Blackcloud P. Psoriasis in skin of color: epidemiology, genetics, clinical presentation, and treatment nuances. J Clin Aesthet Dermatol. 2014;7:16-24.

26. Alinaghi F, Calov M, Kristensen LE, et al. Prevalence of psoriatic arthritis in patients with psoriasis: a systematic review and meta-analysis of observational and clinical studies. J Am Acad Dermatol 2019;80:251-65.e19.
27. Ashman JJ, Hing E, Talwalkar A. Variation in physician office visit rates by patient charateristics and state, 2012. 2015. https://www.cdc.gov/nchs/ products/databriefs/db212.htm. Accessed 8 Jun 2021.

28. Kavanaugh A, Singh R, Karki C, et al. Disease activity and biologic use in patients with psoriatic arthritis or rheumatoid arthritis. Clin Rheumatol. 2018;37:2275-80.

29. Centers for Medicare \& Medicaid Services. Medicare offers more health coverage choices and decreased premiums in 2018. 2017. https://www.cms.gov/ newsroom/press-releases/medicare-offers-morehealth-coverage-choices-and-decreased-premiums2018.

30. U.S. Department of Health and Human Services. Health care coverage analyses of the 2006 national healthcare quality and disparities reports. 2008. https://www.medicaid.gov/medicaid/quality-ofcare/downloads/health-coverage-analyses-of-nhqr. pdf. Accessed 4 Mar 2021.

31. Han X, Call KT, Pintor JK, Alarcon-Espinoza G, Simon AB. Reports of insurance-based discrimination in health care and its association with access to care. Am J Public Health. 2015;105(Suppl 3): S517-25.

32. Churchwell K, Elkind MSV, Benjamin RM, et al. Call to action: structural racism as a fundamental driver of health disparities: a presidential advisory from the American Heart Association. Circulation. 2020;142:e454-68.

33. Dovey ZS, Nair SS, Chakravarty D, Tewari AK. Racial disparity in prostate cancer in the African American population with actionable ideas and novel immunotherapies. Cancer Rep (Hoboken). 2021: e1341.

34. Peek ME, Simons RA, Parker WF, Ansell DA, Rogers SO, Edmonds BT. COVID-19 among African Americans: an action plan for mitigating disparities. Am J Public Health. 2021;111:286-92.

35. Jamalyaria F, Ward MM, Assassi S, et al. Ethnicity and disease severity in ankylosing spondylitis a cross-sectional analysis of three ethnic groups. Clin Rheumatol. 2017;36:2359-64.

36. Mauvais-Jarvis F, Bairey Merz N, Barnes PJ, et al. Sex and gender: modifiers of health, disease, and medicine. Lancet. 2020;396:565-82. 